\begin{tabular}{|c|c|c|}
\hline 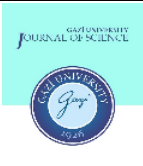 & $\begin{array}{c}\text { Gazi University } \\
\text { Journal of Science }\end{array}$ & 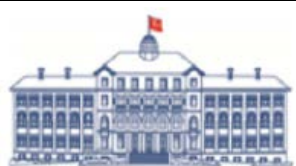 \\
\hline & http://dergipark.gov.tr/gujs & \\
\hline
\end{tabular}

\title{
Geometric-Zero Truncated Poisson Distribution: Properties and Applications
}

\author{
Yunus AKDOGAN ${ }^{1, *}$, , Coskun KUS $^{1}$, Hamid BIDRAM $^{2}$ (D) Ismail KINACI $^{3}$ \\ ${ }^{1}$ Selcuk University, Department of Statistics, Konya, Turkey \\ ${ }^{2}$ University of Isfahan, Department of Statistics, Isfahan, Iran \\ ${ }^{3}$ Selcuk University, Department of Actuary, Konya, Turkey
}

Article Info

Received: $21 / 10 / 2018$

Accepted: 16/03/2019

\section{Keywords}

Compounding

Estimation

Geometric distribution

Zero truncated Poisson

distribution

\begin{abstract}
In this paper, a new discrete distribution is introduced by compounding the geometric distribution with a zero truncated Poisson distribution, named geometric-zero truncated Poisson (GZTP) distribution. Some basic properties of the new distribution, such as the hazard rate function, moments, mode, median, etc., are studied. We show mathematically and numerically that the hazard rate function is increasing. The model parameters are estimated by the moment, least squared error and maximum likelihood methods. A simulation study is performed to compare the performance of the different estimators in terms of bias and mean squared error. An application of the new model is also illustrated using the three real data sets.
\end{abstract}

\section{INTRODUCTION}

In recent years, several new continuous distributions have been introduced by compounding an absolutely continuous distribution with a discrete distribution in the literature. For example, exponential-geometric (Adamidis and Loukas [1]), Weibull-geometric (Barreto-Souza et al.[2]), Weibull-Poisson (Hemmati et al. [3]; Lu and Shi [4]), exponential-Poisson (Kuş [5]), exponential-logarithmic (Tahmasbi and Rezaei [6]) are some remarkable distributions in this connection. Unlike continuous compound distributions, which are created by compounding an absolutely continuous distribution with a discrete one, discrete compound distributions, i.e., compounding two discrete distributions, have not received much attention in the literature, especially in reliability context and statistical modelling.

There exist some complex systems in reliability having components with discrete distributions (see, e.g., Kemp [7] and Noughabi et al. [8]). Now, if the components of a system are themselves random variables with a discrete distribution, then compounding these two discrete distributions can be applied to the lifetime of parallel or series systems. Indeed, the distribution of maximum (minimum) of $N$ components can be obtained by compounding method and has many applications in parallel (series) systems in reliability. Some studies in these subjects can be addressed as uniform-geometric distribution of Akdoğan et al. [9] and uniform-Poisson distribution of Gomez-Deniz [10]. In this paper, we are going to introduce a new discrete distribution by compounding a geometric distribution with a zero-truncated Poisson distribution. The new two-parameter discrete distribution has an increasing hazard rate function, which can be used in modeling discrete real data. This property is proved mathematically under a theorem.

The paper is organized as follows. Section 2 introduces the proposed discrete distribution with its properties, such as probability mass function (pmf), cumulative distribution function (cdf), hazard rate 
and quantile functions as well as median and mode. Section 3 involves moments. The statistical inference is discussed in Section 4. A simulation study is performed in Section 5. Finally, an application of the new discrete model is illustrated in Section 6. Concluding remarks are given in Section 7.

\section{PROPOSED DISCRETE DISTRIBUTION AND ITS PROPERTIES}

\subsection{Letters Pmf and Cdf of the Proposed Distribution}

Let $Y_{1}, Y_{2}, \ldots, Y_{N}$ be $N$ independent identically distributed (iid) random variables having a geometric distribution with the pmf:

$$
P(Y=y)=p q^{y-1} ; \quad y=1,2, \ldots, \quad(0<q=1-p<1),
$$

and $N$ be a zero-truncated Poisson random variable, independent of $Y$, with the pmf:

$$
P(N=n)=\frac{e^{-\lambda} \lambda^{n}}{n !\left(1-e^{-\lambda}\right)} ; \quad n=1,2, \ldots \quad(\lambda>0) .
$$

Now, let us define a random variable as $X=\max \left(Y_{1}, Y_{2}, \ldots, Y_{N}\right)$. Then, its corresponding pmf is obtained by

$$
\begin{aligned}
P_{x}=P(X=x) & =\sum_{n=1}^{\infty} P(X=x \mid N=n) P(N=n) \\
& =\sum_{n=1}^{\infty}\left(\left(1-q^{x}\right)^{n}-\left(1-q^{x-1}\right)^{n}\right) \frac{e^{-\lambda} \lambda^{n}}{n !\left(1-e^{-\lambda}\right)} \\
& =\frac{e^{-\lambda}}{1-e^{-\lambda}}\left(\sum_{n=1}^{\infty} \frac{\left(\lambda\left(1-q^{x}\right)\right)^{n}}{n !}-\sum_{n=1}^{\infty} \frac{\left(\lambda\left(1-q^{x-1}\right)\right)^{n}}{n !}\right) \\
& = \begin{cases}\frac{1}{1-\theta}\left(\theta^{q^{x}}-\theta^{x^{x-1}}\right) ; & x=1,2, \ldots \\
0 ; & \text { otherwise, }\end{cases}
\end{aligned}
$$

where $\theta=e^{-\lambda} \in(0,1)$. The cdf of $X$ is obtained by

$$
\begin{aligned}
F_{X}(x)=P(X \leq x) & =\sum_{n=1}^{\infty} P(X \leq x \mid N=n) P(N=n) \\
& =\sum_{n=1}^{\infty}\left(1-q^{x}\right)^{n} \frac{e^{-\lambda} \lambda^{n}}{n !\left(1-e^{-\lambda}\right)} \\
& =\frac{e^{-\lambda q^{x}}-e^{-\lambda}}{1-e^{-\lambda}} \\
& =\frac{\theta^{q^{x}}-\theta}{1-\theta} ; \quad x=1,2, \ldots,
\end{aligned}
$$

whose final form is given by

$$
F(x)= \begin{cases}0 ; & x \leq 0 \\ \frac{\theta^{9^{x}}-\theta}{1-\theta} ; & x=1,2, \ldots\end{cases}
$$

The random variable $X$ with the pmf Eq. (2) is said to have a geometric-zero truncated Poisson 
distribution and will be denoted by $X \sim \operatorname{GZTP}(q, \theta)$. The random variable $X$ is potentially useful in various fields, especially in parallel systems considering discrete distribution for their components (see, e.g., Nakagawa and Zhao [11]).

Figure 1 shows the pmfs of the $\operatorname{GZTP}(q, \theta)$ distribution for some parameter values of $q$ and $\theta$. As we see from the graphs, for fixed values of $q$, the $\operatorname{pmf}$ of the $\operatorname{GZTP}(q, \theta)$ distribution varies from increasingdecreasing to decreasing, when the parameter $\theta$ increases.
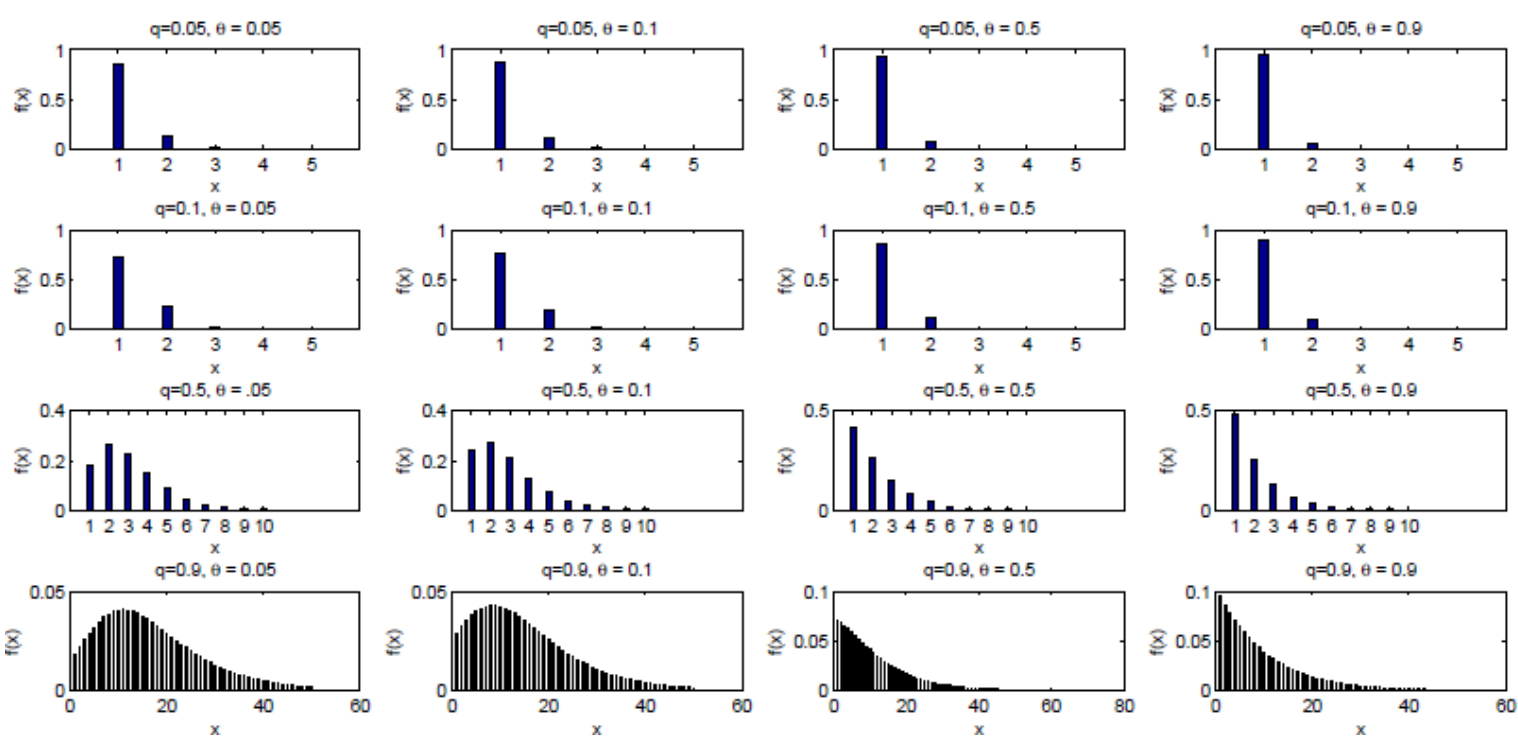

Figure 1. The pmfs of $\operatorname{GZTP}(q, \theta)$ distribution for different values of $q$ and $\theta$

Theorem 2.1. The pmf of $\operatorname{GZTP}(q, \theta)$ distribution is log-concave for any admissible value of $q$ and $\theta$.

Proof. We should show that $P_{x}^{2} \geq P_{x-1} P_{x+1}$ Keilson and Gerber [12]. Using pmf given in Eq. (3) we write $\left(\theta^{q^{x}}-\theta^{q^{x-1}}\right)^{2} \geq\left(\theta^{q^{x-1}}-\theta^{q^{x-2}}\right)\left(\theta^{q^{x+1}}-\theta^{q^{x}}\right)$.

Now, consider the function $g(x ; q, \theta)=\left(\theta^{q^{x}}-\theta^{q^{x-1}}\right)^{2}-\left(\theta^{q^{x-1}}-\theta^{q^{x-2}}\right)\left(\theta^{\theta^{x^{x+1}}}-\theta^{q^{x}}\right)$. Then, one can show that $g(x ; q, \theta)$ is decreasing for all $x \in R^{+}$. In addition, it is clear that $\lim _{x \rightarrow \infty} g(x ; q, \theta)=0$. Therefore, $g(x ; q, \theta)$ is a non-negative function and then the proof is complete.

\section{Corollary(Unimodal):}

The pmf of $\operatorname{GZTP}(q, \theta)$ distribution is unimodal and its mode is

$\bmod (X)=\left\{\begin{array}{cc}\lfloor m\rfloor ; & f(\lfloor m\rfloor)>f(\mid m\rfloor+1) \\ \lfloor m\rfloor+1 ; & f(\lfloor m\rfloor)<f(\mid m\rfloor+1) \\ \{[m],[m]+1\} ; & f([m])=f([m]+1),\end{array}\right.$

where $m=\log \left(\frac{\log (q)}{(1-q) \log \theta}\right) / \log (q)$ and $|x|$ denotes the integer part of $x$. 
Proof. The unimodality of $\operatorname{GZTP}(q, \theta)$ distribution is achieved by the fact that log-concave pmfs are strongly unimodal and thus unimodal. (see, e.g., Keilson and Gerber [12]) To obtain $\bmod (X)$, let us consider the pmf given in (pmf) be a continuous function in $X$. Then, it is easy to see that the maximum of the pmf happens at the point $m=\log \left(\frac{\log (q)}{(1-q) \log \theta}\right) / \log (q)$.

\subsection{Hazard Rate Function}

Let $X \sim \operatorname{GZTP}(q, \theta)$. Then, the hazard rate function of $X$ is given by

$$
\begin{aligned}
h_{0}(x) & =P(X=x \mid x) \\
& =\frac{P(X=x)}{P(X \geq x)} \\
& =\frac{\theta^{q^{x}}-\theta^{q^{x-1}}}{1-\theta^{q^{x-1}}} ; \quad x=1,2, \ldots
\end{aligned}
$$

Theorem 2.2. The hazard rate function of $\operatorname{GZTP}(q, \theta)$ distribution is increasing for any value of $q$ and $\theta$.

Proof. It is obvious that log-concave pmfs have an increasing hazard rate function (see, e.g., Keilson and Gerber [12]). Thus, using Theorem 2.1, the hazard rate function is increasing for any value of $q$ and $\theta$. The plots of hazard rate function are given in Figure 2. As we see from the graph, the hazard rate function of $\operatorname{GZTP}(q, \theta)$ is increasing for all values of $q$ and $\theta$ parameters.
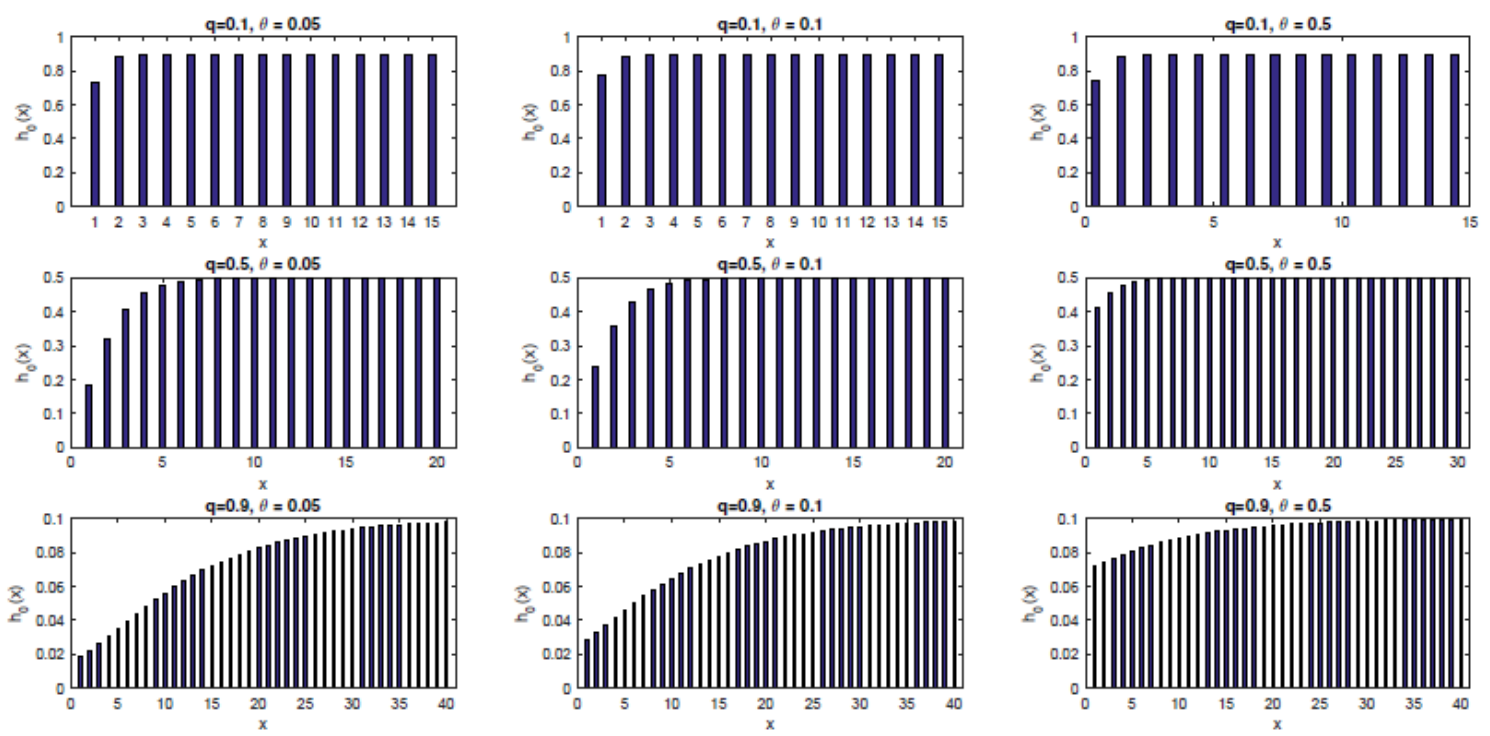

Figure 2. The hazard rate functions of $\operatorname{GZTP}(q, \theta)$ for different values of $q$ and $\theta$

\subsection{Quantile Function}

The quantile function of the $\operatorname{GZTP}(q, \theta)$ distribution, say $Q(u)$, is obtained by $F(Q(u))=u$. Then, using Eq. (3), we have: 
$\frac{\theta^{Q^{(u)}}-\theta}{1-\theta}=u$

Inverting Eq.(4), one obtains

$Q(u)=\frac{\log \left(u \theta^{-1}-u+1\right)}{\log (q)}$.

From the non-decreasing property of $Q(u)$, the $z^{\text {th }}$ quantile $\left(x_{z}\right)$, of $\operatorname{GZTP}(q, \theta)$ distribution is given by

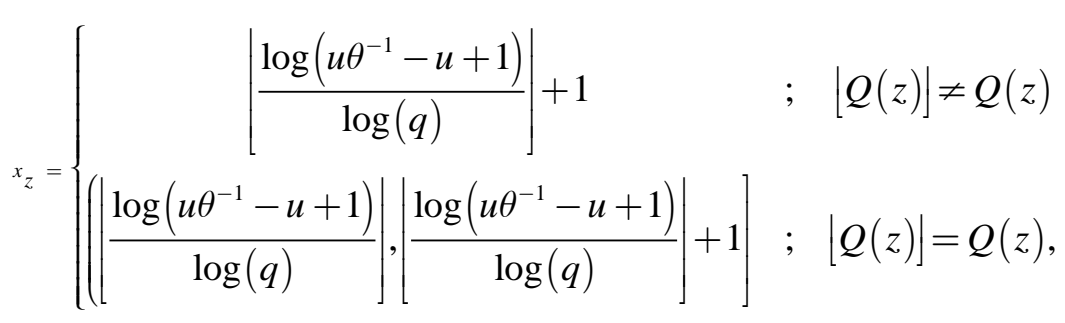

where $\left\lfloor x \mid\right.$ denotes the integer part of $x$. That is $x_{z}$ satisfies $F\left(x_{z}^{-}\right) \leq p \leq F\left(x_{z}\right)$, where $F$ is the cdf of $\operatorname{GZTP}(q, \theta)$ distribution given in Eq.(3). In a special case, the median of $\operatorname{GZTP}(q, \theta)$ distribution is also given by

$$
x_{0.5}=\left\{\begin{array}{cc}
\left\lfloor\frac{\log \left(0.5 \theta^{-1}+0.5\right)}{\log (q)} \mid+1\right. & ;|Q(0.5)| \neq Q(0.5) \\
\left(\left|\frac{\log \left(0.5 \theta^{-1}+0.5\right)}{\log (q)}\right|,\left|\frac{\log \left(0.5 \theta^{-1}+0.5\right)}{\log (q)}\right|+1\right] & ; \quad|Q(0.5)|=Q(0.5) .
\end{array}\right.
$$

\section{MOMENTS}

\subsection{Approximate and Exact Bounds of the Moments}

Let $X \sim \operatorname{GZTP}(q, \theta)$. Then the expected value is calculated by

$E(X)=\frac{1}{1-\theta} \sum_{x=1}^{\infty} X\left(\theta^{q^{x}}-\theta^{q^{x-1}}\right)$.

It is clear that $E(X)$ can not be calculated easily using the above equation. Therefore, we attempt to discuss an approximate value for $E(X)$ here. A method for estimating the sum of a positive series, whose convergence has been guaranteed, is the ratio test method. Let $S=\sum_{n=1}^{\infty} a_{n}$ and $S_{n}=\sum_{k=1}^{n} a_{k}$ be the $n$th partial sum. The ratio test method is given in the following lemma.

Lemma 1. (The ratio test; Braden [13]). Suppose $\left\{a_{n}\right\}$ is a positive decreasing sequence such that $\lim _{n \rightarrow \infty} \frac{a_{n+1}}{a n}=L<1$. If $\frac{a_{n+1}}{a n}$ decreases to $L$, then 
$S_{n}+a_{n}\left(\frac{L}{1-L}\right)<S<S_{n}+\frac{a_{n+1}}{1-\frac{a_{n+1}}{a_{n}}}$.

Now, we discuss the expected value of $\operatorname{GZTP}(q, \theta)$ distribution in the following:

$$
\begin{aligned}
E(X) & =\frac{1}{1-\theta} \sum_{x=1}^{\infty} x\left(\theta^{q^{x}}-\theta^{x^{x-1}}\right) \\
& =\frac{\sum_{x=1}^{c} a_{x}+\sum_{x=c+1}^{\infty} a_{x}}{1-\theta} \\
& =\frac{\sum_{x=1}^{c} a_{x}+\sum_{y=1}^{\infty} a_{y+c}}{1-\theta},
\end{aligned}
$$

where $a_{x}=x\left(\theta^{q^{x}}-\theta^{q^{x-1}}\right)$ and $c=\left|\arg \max \left(a_{x}\right)\right|$. From Theorem 2.1, it is clear that $a_{x}$ is unimodal. Furthermore $a_{x}$ is decreasing in $x$ for $x \geq c$. By using ratio test method, one can obtain

$$
\frac{\frac{q}{1-q} a_{n+c}+\sum_{x=1}^{n+c} a_{x}}{1-\theta} \leq E(X) \leq \frac{\frac{a_{n+c+1}}{1-\frac{-\frac{a_{n++1}}{a_{n+c}}}{1-\theta}}+\sum_{x=1}^{n+c} a_{x}}{1-\theta},
$$

since $\left\{a_{n} ; n \geq c\right\}$ is positive decreasing sequence, $\lim _{n \rightarrow \infty} \frac{a_{n+c+1}}{a_{n+c}}=q<1$ and $\frac{a_{n+c+1}}{a_{n+c}}$ decreases to $q$. The other moments can be obtained similarly. Table 1 contain first moment of $\operatorname{GZTP}(q, \theta)$ distribution for some parameter values. The true values of moments are provided by Maple software. Some approximations of moments are also presented for $\theta=e^{-5}$ and $q=0.5$ in Figure 3. From Tables 1-4, all approximations are good enough $n \geq 150$ for selected cases.

Table 1. Approximate value, lower bound and upper bound of $E(X)$

\begin{tabular}{|l|l|l|l|l|l|}
\hline$(q, \theta)$ & $E(X)$ & $n$ & App. of $E(X)$ & Lower Bound of $E(X)$ & Upper Bound of $E(X)$ \\
\hline \multirow{3}{*}{$\left(0.3, e^{-2}\right)$} & 1.7997151003 & 5 & 1.7993766884 & 1.7989899838 & 1.7997633932 \\
\cline { 2 - 6 } & & 10 & 1.7997142548 & 1.7997133442 & 1.7997151653 \\
\cline { 2 - 6 } & & 20 & 1.7997151003 & 1.7997151003 & 1.7997151003 \\
\hline \multirow{3}{*}{$\left(0.5, e^{-2}\right)$} & 2.7194339620 & 5 & 2.7116771654 & 2.7008500136 & 2.7225043172 \\
\cline { 2 - 6 } & & 10 & 2.7191750259 & 2.7188684061 & 2.7194816456 \\
\cline { 2 - 6 } & & 20 & 2.7194336988 & 2.7194334105 & 2.7194339870 \\
\cline { 2 - 6 }$\left(0.8, e^{-2}\right)$ & 7.3433586356 & 5 & 7.2709297316 & 6.6218080726 & 7.9200513908 \\
\cline { 2 - 6 } & & 10 & 7.2760098324 & 7.1206461299 & 7.4313735348 \\
\cline { 2 - 6 } & & 20 & 7.3340874125 & 7.3207103315 & 7.3474644935 \\
\cline { 2 - 6 } & & 150 & 7.3433586356 & 7.3433586356 & 7.3433586356 \\
\hline \multirow{2}{*}{$\left(0.3, e^{-5}\right)$} & 2.3314642857 & 5 & 2.3312413457 & 2.3309910793 & 2.3314916121 \\
\hline
\end{tabular}




\begin{tabular}{|l|l|l|l|l|l|}
\hline & & 10 & 2.3314637320 & 2.3314631392 & 2.3314643248 \\
\cline { 2 - 6 } & & 20 & 2.3314642857 & 2.3314642857 & 2.3314642857 \\
\hline \multirow{3}{*}{$\left(0.5, e^{-5}\right)$} & 3.6795124171 & 5 & 3.6708662414 & 3.6590567668 & 3.6826757160 \\
\cline { 2 - 6 } & & 10 & 3.6792286275 & 3.6788966754 & 3.6795605796 \\
\cline { 2 - 6 } & & 20 & 3.6795121301 & 3.6795118170 & 3.6795124432 \\
\hline \multirow{3}{*}{$\left(0.8, e^{-5}\right)$} & 10.3715943115 & 5 & 10.2727957683 & 9.5103299111 & 11.0352616212 \\
\cline { 2 - 6 } & & 10 & 10.2921570783 & 10.1152364277 & 10.4690777314 \\
\cline { 2 - 6 } & & 20 & 10.3610415078 & 10.3462252766 & 10.3758577390 \\
\cline { 2 - 6 } & & 150 & 10.3715943115 & 10.3715943115 & 10.3715943115 \\
\hline
\end{tabular}
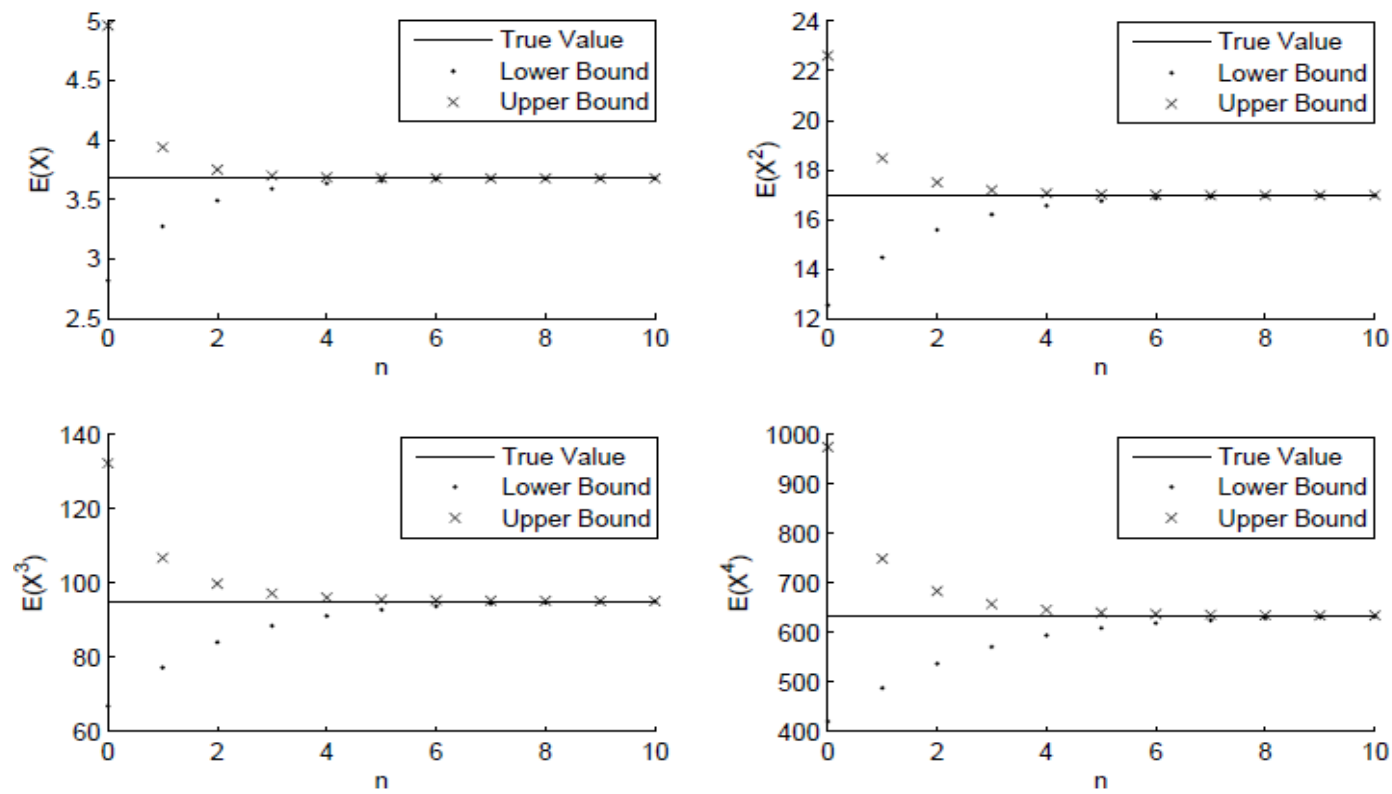

Figure 3. Some approximate moments of $\operatorname{GZTP}(q, \theta)$ for $q=0.5$ and $\theta=\exp (-5)$

\subsection{Approximate and Exact Variance, Skewness, and Kurtosis}

In this section, approximate and exact variance, skewness and kurtosis of $\operatorname{GTPP}(q, \theta)$ are given in

Tables 2-4.

Table 2. Exact and approximate variance for some parameter values of $q$ and $\theta$

\begin{tabular}{|l|l|l|l|l|l|l|l|}
\hline$(q, \theta)$ & $\operatorname{Var}(X)$ & $n$ & Appr. Var $(X)$ & $(q, \theta)$ & $\operatorname{Var}(X)$ & $n$ & Appr. Var $(X)$ \\
\hline$\left(0.3, e^{-2}\right)$ & 0.9668832158 & 5 & 0.9663090140 & $\left(0.3, e^{-5}\right)$ & 1.1907613964 & 5 & 1.1878995568 \\
\hline & & 10 & 0.9668792184 & & & 10 & 1.1907486552 \\
\hline & & 20 & 0.9668832158 & & & 20 & 1.1907613964 \\
\hline$\left(0.5, e^{-2}\right)$ & 2.9317233071 & 5 & 2.8966614573 & $\left(0.5, e^{-5}\right)$ & 3.4392247529 & 5 & 3.4078153711 \\
\hline & & 20 & 2.9317180597 & & & 20 & 3.4392192935 \\
\hline & & 50 & 2.9317233071 & & & 50 & 3.4392247529 \\
\hline
\end{tabular}




\begin{tabular}{|l|l|l|l|l|l|l|l|}
\hline$\left(0.8, e^{-2}\right)$ & 28.2784602533 & 5 & 27.9418642698 & $\left(0.8, e^{-5}\right)$ & 32.5653946091 & 5 & 33.2237469999 \\
\hline & & 20 & 28.1456910482 & & & 20 & 32.2997608568 \\
\hline & & 50 & 28.2779548653 & & & 50 & 32.5644642142 \\
\hline & & 150 & 28.2784602533 & & & 150 & 32.5653946091 \\
\hline
\end{tabular}

Table 3. Exact and approximate Skewness for some parameter values of $q$ and $\theta$

\begin{tabular}{|l|l|l|l|l|l|l|l|}
\hline$(q, \theta)$ & Skewness $(X)$ & $n$ & Appr. Skewness $(X)$ & $(q, \theta)$ & Skewness $(X)$ & $n$ & Appr. Skewness $(X)$ \\
\hline$\left(0.3, e^{-2}\right)$ & 1.5439885033 & 5 & 1.5293884914 & $\left(0.3, e^{-5}\right)$ & 1.0913310183 & 5 & 1.0974467318 \\
\hline & & 10 & 1.5438744811 & & & 10 & 1.0913286139 \\
\hline & & 20 & 1.5439885033 & & & 20 & 1.0913310183 \\
\hline$\left(0.5, e^{-2}\right)$ & 1.4760392637 & 5 & 1.4620813735 & $\left(0.5, e^{-5}\right)$ & 1.1516492654 & 5 & 1.1686130051 \\
\hline & & 20 & 1.4760226997 & & & 20 & 1.1516370129 \\
\hline & & 50 & 1.4760392637 & & & 50 & 1.1516492654 \\
\hline$\left(0.8, e^{-2}\right)$ & 1.4565690343 & 5 & 1.6365220116 & $\left(0.8, e^{-5}\right)$ & 1.1788315851 & 5 & 1.3763783197 \\
\hline & & 20 & 1.4392972052 & & & 20 & 1.1750124067 \\
\hline & & 50 & 1.4564145782 & & & 50 & 1.1786652455 \\
\hline & & 150 & 1.4565690343 & & & 150 & 1.1788315851 \\
\hline
\end{tabular}

Table 4. Exact and approximate kurtosis for some parameter values of $q$ and $\theta$

\begin{tabular}{|l|l|l|l|l|l|l|l|}
\hline$(q, \theta)$ & Kurtosis $(X)$ & $n$ & Appr. Kurtosis $(X)$ & $(q, \theta)$ & Kurtosis $(X)$ & $n$ & Appr. Kurtosis $(X)$ \\
\hline$\left(0.3, e^{-2}\right)$ & 6.3909103067 & 5 & 6.4108993095 & $\left(0.3, e^{-5}\right)$ & 5.1377052475 & 5 & 4.9970349940 \\
\hline & & 10 & 6.3908145204 & & & 10 & 5.1365329801 \\
\hline & & 20 & 6.3909103066 & & & 20 & 5.1377052475 \\
\hline$\left(0.5, e^{-2}\right)$ & 6.3265775593 & 5 & 6.2994312225 & $\left(0.5, e^{-5}\right)$ & 5.3682140159 & 5 & 5.0740361546 \\
\hline & & 20 & 6.3265408209 & & & 20 & 5.3680842138 \\
\hline & & 50 & 6.3265775593 & & & 50 & 5.3682140159 \\
\hline$\left(0.8, e^{-2}\right)$ & 6.3183347918 & 5 & 6.2224533983 & $\left(0.8, e^{-5}\right)$ & 5.4654383959 & 5 & 4.8882486478 \\
\hline & & 20 & 6.2211167752 & & & 20 & 5.3577364298 \\
\hline & & 50 & 6.3173067981 & & & 50 & 5.4644336039 \\
\hline & & 150 & 6.3183347918 & & & 150 & 5.4654383959 \\
\hline
\end{tabular}

From Tables 3 and 4, it seems that $\operatorname{GZTP}(q, \theta)$ is rightly-skewed and leptokurtic.

\section{PARAMETER ESTIMATION}

\subsection{Estimation by the Maximum Likelihood Method}

Let $X_{1}, X_{2}, \ldots, X_{n}$ be a complete random sample from $\operatorname{GZTP}(q, \theta)$ distribution. The likelihood and 
log-likelihood functions based on the complete random sample are

$L(q, \theta)=\prod_{i=1}^{n} \frac{1}{1-\theta}\left(\theta^{q^{x_{i}}}-\theta^{q^{x_{i}-1}}\right)$

and

$\ell(q, \theta)=-n \log (1-\theta)+\sum_{i=1}^{n} \log \left(\theta^{\theta^{\alpha_{i}}}-\theta^{\alpha^{x_{i}-1}}\right)$,

respectively. Thus, the score equations are obtained by

$$
\begin{aligned}
& \frac{\partial \ell(q, \theta)}{\partial q}=\sum_{i=1}^{n}\left(\frac{\theta^{q^{x_{i}}} x_{i} q^{\alpha_{i}-1} \log \theta-\theta^{q^{x_{i}-1}}\left(x_{i}-1\right) q^{x_{i}-2} \log \theta}{\theta^{q_{i}}-\theta^{\alpha_{i}-1}}\right)=0 \\
& \frac{\partial \ell(q, \theta)}{\partial \theta}=\frac{n}{1-\theta}+\sum_{i=1}^{n} \frac{q^{x_{i}} \theta^{q^{\alpha_{i}}-1}-q^{x_{i}-1} \theta^{q^{\alpha_{i}-1}-1}}{\theta^{q^{x_{i}}}-\theta^{q^{q_{i}-1}}}=0 .
\end{aligned}
$$

The maximum likelihood estimation (MLE) of the parameters, i.e., $\hat{q}$ and $\hat{\theta}$ can be achieved by solving Eqs (7) and (8), using Newton-Raphson procedure. An approximate Fisher information matrix can be obtained by

$$
I(\hat{q}, \hat{\theta}) \approx\left[\begin{array}{cc}
-\left.\frac{\partial^{2} \ell(q, \theta)}{\partial q^{2}}\right|_{(\hat{q}, \hat{\theta})} & -\left.\frac{\partial^{2} \ell(q, \theta)}{\partial q \partial \theta}\right|_{(\hat{q}, \hat{\theta})} \\
-\left.\frac{\partial^{2} \ell(q, \theta)}{\partial \theta \partial q}\right|_{(\hat{q}, \hat{\theta})} & -\left.\frac{\partial^{2} \ell(q, \theta)}{\partial \theta^{2}}\right|_{(\hat{q}, \hat{\theta})}
\end{array}\right],
$$

whose entries are the estimated second order derivatives of Eq. (6). It can be shown that the $\operatorname{GZTP}(q, \theta)$ family satisfies the regularity conditions which are fulfilled for parameters in the interior of the parameter space but not on the boundary (see, e.g., Ferguson [14]). Thus, $I^{\frac{1}{2}}(\hat{q}, \hat{\theta})\left[(\hat{q}, \hat{\theta})^{T}-(q, \theta)^{T}\right]$ converges in distribution to the bivariate standard normal. Now, approximat $100(1-\alpha) \%$ confidence intervals for the parameters are $\left(\hat{q}-z_{\frac{\alpha}{2}} \sqrt{V_{11}}, \hat{q}+z_{\frac{\alpha}{2}} \sqrt{V_{11}}\right)$ and $\left(\hat{\theta}-z_{\frac{\alpha}{2}} \sqrt{V_{22}}, \hat{\theta}+z_{\frac{\alpha}{2}} \sqrt{V_{22}}\right)$, where $V_{11}$ and $V_{22}$ are the elements on the main diagonal of the covariance matrix $I^{-1}(\hat{q}, \hat{\theta})$ and $z_{\frac{\alpha}{2}}$ is the percentile of the standard normal distribution with right-tail probability $\alpha / 2$.

\subsection{Estimation by the Method of Moments}

To estimate the parameters of $\operatorname{GZTP}(q, \theta)$ distribution by the method of moments (MM), we need the first and second sample moments, which are given below:

$$
\begin{aligned}
& \frac{1}{1-\theta} \sum_{x=1}^{\infty} x\left(\theta^{q^{x}}-\theta^{q^{x-1}}\right)=\frac{1}{n} \sum_{i=1}^{n} X_{i}, \\
& \frac{1}{1-\theta} \sum_{x=1}^{\infty} x^{2}\left(\theta^{q^{x}}-\theta^{q^{x-1}}\right)=\frac{1}{n} \sum_{i=1}^{n} X_{i}^{2} .
\end{aligned}
$$


Eqs (10) and (11) can be solved numerically using Newton-Raphson method. The solutions of eqs (10) and (11) are moments estimates $(\tilde{q}, \tilde{\theta})$ of parameters $(q, \theta)$.

\subsection{Estimation by Least Squares Error Method}

Let $x_{(1)}<x_{(2)}<\cdots<x_{(n)}$ denote the ordered observations from $\operatorname{GZTP}(q, \theta)$ distribution. Using the distribution function given in Eq. (3), we have

$$
F\left(x_{(i)}\right)=\frac{\theta^{q^{x_{i}}}-\theta}{1-\theta} ; \quad i=1,2, \ldots, n .
$$

Empirical distribution function, denoted by $F^{*}$, can be used to estimate $F\left(x_{(i)}\right)$. Substituting the empirical distribution function in Eq. (12), we have the following model:

$F^{*}\left(x_{(i)}\right)=\frac{\theta^{q^{x_{i}}}-\theta}{1-\theta}+\varepsilon_{i} ; \quad i=1,2, \ldots, n$,

where $\varepsilon_{i}$ is the error term for $i$ th observation. Now, least squares error (LSE) estimators $\left(\hat{q}_{*}, \hat{\theta}_{*}\right)$ of the parameters can be obtained by minimizing the following equation with respect to $q$ and $\theta$ :

$L(q, \theta)=\sum_{i=1}^{n} \varepsilon_{i}^{2}=\sum_{i=1}^{n}\left(F^{*}\left(x_{(i)}\right)-\frac{\theta^{q^{x_{i}}}-\theta}{1-\theta}\right)^{2} ; \quad i=1,2, \ldots, n$.

This procedure can be performed by Gauss-Newton method.

\section{SIMULATION STUDY}

In this section, a simulation study is performed to compare the performance of different estimations discussed in the last section. In this simulation, we generate 10000 random samples with sizes 50, 100, 300, and 500 from the $\operatorname{GZTP}(q, \theta)$ distribution and then compute the MLE, MM and LSE of $q$ and $\theta$. A random number from $\operatorname{GZTP}(q, \theta)$ can be generated by using the following algorithm:

A1. Generate $N \sim \operatorname{ZTP}(\lambda)$

A2. Generate $Y_{1}, Y_{2}, \ldots, Y_{N} \sim \operatorname{iidGeo}(q)$

A3. Calculate $X=\max \left(Y_{1}, Y_{2}, \ldots, Y_{N}\right)$. Then $\left(X_{1}, X_{2}, \ldots, X_{n}\right)$ required sample from the $\operatorname{GZTP}(q, \theta)$ distribution.

We compare the performance of these estimators in terms of their biases and mean square errors (MSEs). In Tables 5 and 6, we report the biases and MSEs of these estimators for some parameter values. 
Table 5. Biases of MLE, MM and LSE estimators for some parameter values of $q$ and $\theta$

\begin{tabular}{|l|l|l|l|l|l|l|l|}
\hline & \multicolumn{3}{|l}{ MLE } & MM & \multicolumn{2}{l|}{ LSE } \\
\hline$(q, \theta)$ & $n$ & $\hat{q}$ & $\hat{\theta}$ & $\tilde{q}$ & $\tilde{\theta}$ & $\hat{q}_{*}$ & $\hat{\theta}_{*}$ \\
\hline \multirow{5}{*}{$\left(0.2, e^{-2}\right)$} & 50 & 0.0049 & 0.1418 & 0.0094 & 0.3012 & 0.0062 & 0.1792 \\
\cline { 2 - 8 } & 100 & 0.0031 & 0.0744 & 0.0055 & 0.1583 & 0.0034 & 0.9998 \\
\cline { 2 - 8 } & 300 & 0.0009 & 0.0285 & 0.0016 & 0.0514 & 0.0010 & 0.0328 \\
\cline { 2 - 8 } & 500 & 0.0005 & 0.0121 & 0.0009 & 0.0279 & 0.0004 & 0.0132 \\
\hline \multirow{5}{*}{$\left(0.3, e^{-5}\right)$} & 50 & 0.0056 & 0.3738 & 0.0096 & 0.6610 & 0.0054 & 0.4207 \\
\cline { 2 - 8 } & 100 & 0.0027 & 0.1727 & 0.0047 & 0.3166 & 0.0027 & 0.1967 \\
\cline { 2 - 8 } & 300 & 0.0008 & 0.0504 & 0.0015 & 0.0987 & 0.0008 & 0.0604 \\
\cline { 2 - 8 } & 500 & 0.0005 & 0.0293 & 0.0008 & 0.0541 & 0.0003 & 0.0286 \\
\hline \multirow{5}{*}{$\left.0.5, e^{-2}\right)$} & 50 & 0.0037 & 0.0942 & 0.0115 & 0.2540 & -0.0003 & 0.0583 \\
\cline { 2 - 8 } & 100 & 0.0023 & 0.0693 & 0.0071 & 0.1622 & 0.0008 & 0.0577 \\
\cline { 2 - 8 } & 300 & 0.0019 & 0.0370 & 0.0034 & 0.0673 & 0.0016 & 0.0362 \\
\cline { 2 - 8 } & 500 & 0.0003 & 0.0151 & 0.0013 & 0.0342 & 0.0002 & 0.0146 \\
\hline \multirow{5}{*}{$\left(0.7, e^{-3}\right)$} & 50 & 0.0041 & 0.3270 & 0.0117 & 0.5562 & -0.0016 & 0.2687 \\
\cline { 2 - 8 } & 100 & 0.0034 & 0.1880 & 0.0072 & 0.3040 & 0.0012 & 0.1668 \\
\cline { 2 - 7 } & 300 & 0.0005 & 0.0465 & 0.0018 & 0.0859 & 0.0004 & 0.0427 \\
\cline { 2 - 8 } & 500 & 0.0005 & 0.0306 & 0.0014 & 0.0535 & 0.0001 & 0.0294 \\
\hline
\end{tabular}

Table 6. MSEs of MLE, MM and LSE estimators for some parameter values of $q$ and $\theta$

\begin{tabular}{|l|l|l|l|l|l|l|l|}
\hline & \multicolumn{3}{|l}{ MLE } & MM & \multicolumn{2}{l|}{ LSE } \\
\hline$(q, \theta)$ & $n$ & $\hat{q}$ & $\hat{\theta}$ & $\tilde{q}$ & $\tilde{\theta}$ & $\hat{q}_{*}$ & $\hat{\theta}_{*}$ \\
\hline \multirow{3}{*}{$\left(0.2, e^{-2}\right)$} & 50 & 0.0010 & 0.8286 & 0.0013 & 1.2071 & 0.0013 & 0.9998 \\
\cline { 2 - 8 } & 100 & 0.0005 & 0.4021 & 0.0007 & 0.5967 & 0.0006 & 0.4606 \\
\cline { 2 - 8 } & 300 & 0.0002 & 0.1195 & 0.0002 & 0.1865 & 0.0002 & 0.1321 \\
\cline { 2 - 8 } & 500 & 0.0001 & 0.0735 & 0.0001 & 0.1119 & 0.0001 & 0.0815 \\
\hline$\left(0.3, e^{-5}\right)$ & 50 & 0.0011 & 2.1478 & 0.0016 & 3.8685 & 0.0013 & 2.9504 \\
\cline { 2 - 8 } & 100 & 0.0005 & 0.8743 & 0.0008 & 1.6046 & 0.0006 & 1.1230 \\
\cline { 2 - 8 } & 300 & 0.0002 & 0.2567 & 0.0003 & 0.4671 & 0.0002 & 0.3235 \\
\cline { 2 - 8 } & 500 & 0.0001 & 0.1475 & 0.0002 & 0.2638 & 0.0001 & 0.1857 \\
\hline \multirow{5}{*}{$\left.0.5, e^{-2}\right)$} & 50 & 0.0042 & 1.1331 & 0.0051 & 1.5048 & 0.0051 & 1.2964 \\
\cline { 2 - 8 } & 100 & 0.0021 & 0.4962 & 0.0026 & 0.6778 & 0.0023 & 0.5323 \\
\cline { 2 - 8 } & 300 & 0.0007 & 0.1529 & 0.0009 & 0.2188 & 0.0007 & 0.1606 \\
\cline { 2 - 8 } & 500 & 0.0004 & 0.0914 & 0.0006 & 0.1325 & 0.0004 & 0.0959 \\
\hline \multirow{5}{*}{$\left.0.7, e^{-3}\right)$} & 50 & 0.0044 & 2.2897 & 0.0052 & 3.0230 & 0.0052 & 2.5288 \\
\cline { 2 - 8 } & 100 & 0.0021 & 0.9256 & 0.0027 & 1.2521 & 0.0024 & 0.9991 \\
\cline { 2 - 8 } & 300 & 0.0007 & 0.2763 & 0.0009 & 0.3710 & 0.0008 & 0.2942 \\
\cline { 2 - 7 } & 500 & 0.0004 & 0.1598 & 0.0005 & 0.2153 & 0.0005 & 0.1708 \\
\hline
\end{tabular}

From Tables 5 and 6, we see that all estimators are biased but asymptotically unbiased. The MLE and the LSE are almost identical in terms of MSE and both performs better than MM. Also, as the sample size $n$ increases, the bias and MSE of the estimators reduce as expected.

\section{APPLICATION}

In this section, we fit the $\operatorname{GZTP}(q, \theta)$ model to the two real data sets and compare it with the following 
models:

1. Discrete Weibull (DW) distribution of Nakagawa and Osaki [15] with the pmf:

$$
f_{D W}(x ; p, \alpha, \gamma)=p^{x^{\alpha}}-p^{(x+1)^{\alpha}} ; x=0,1,2, \ldots, \alpha>0,0<p<1 .
$$

2. Exponentiated discrete Weibull (EDW) of Nekoukhou and Bidram [16] with the pmf:

$$
f_{E D W}(x ; p, \alpha, \gamma)=\left(1-p^{(x+1)^{\alpha}}\right)^{\gamma}-\left(1-p^{x^{\alpha}}\right)^{\gamma} ; x=0,1,2, \ldots, \alpha>0, \gamma>0,0<p<1 .
$$

3. Discrete generalized exponential (DGE) distribution of Nekoukhou et al. [17] with the pmf:

$$
f_{D G E}(x ; \alpha, p)=k p^{x-1}\left(1-p^{x}\right)^{\alpha-1}, \quad x=1,2, \ldots, \alpha>0,0<p<1,
$$

4. Geometric distribution with the pmf:

$f_{G e}(x ; p)=(1-p) p^{x-1}, \quad x=1,2, \ldots, 0<p<1$,

5. Zero-truncated Poisson (ZTP) distribution with the pmf Eq. (2).

6. Discrete Poisson-Lindley (DPL) of Sankaran [18] distribution with the pmf

$$
f_{D P L}(x ; \theta)=\theta^{2}(\theta+x+2) /(\theta+1)^{x+3} ; \quad x=1,2, \ldots, \alpha>0 .
$$

The MLE, maximized log-likelihood, AIC (Akaike Information Criterion) and Kolmogorov-Smirnov (KS) values are calculated for all models.

1. First real data set: Table 7 contains the number of failures in a certain time interval (of equal length) given and analyzed by Xie and Lai [19].

Table 7. The number of failures in a certain time interval (of equal length)

\begin{tabular}{|l|l|l|l|l|l|}
\hline Time & No of failures & Time & No of failures & Time & No of failures \\
\hline 1 & 53 & 7 & 22 & 13 & 13 \\
\hline 2 & 29 & 8 & 16 & 14 & 5 \\
\hline 3 & 29 & 9 & 18 & 15 & 5 \\
\hline 4 & 36 & 10 & 8 & 16 & 4 \\
\hline 5 & 13 & 11 & 22 & 17 & 1 \\
\hline 6 & 25 & 12 & 11 & 18 & 1 \\
\hline
\end{tabular}

The summary of calculations is given in Table 8 .

Table 8. MLE, maximized $\ell, A I C$, and K-S values of the fitted models for the first data set

\begin{tabular}{|l|l|l|l|l|}
\hline Model & MLE & $-\ell(\hat{\boldsymbol{\theta}})$ & AIC & K-S \\
\hline GZTP & $\hat{q}=0.7904, \hat{\theta}=0.3100$ & 835.2550 & 1674.5 & 0.1255 \\
\hline DW & $\hat{p}=0.9579, \hat{\alpha}=1.5861$ & 851.0519 & 1706.1 & 0.4720 \\
\hline EDW & $\hat{p}=0.9581, \hat{\alpha}=1.5881, \hat{\gamma}=0.9979$ & 851.0519 & 1708.1 & 0.1213 \\
\hline DGE & $\hat{\alpha}=1.3715, \hat{p}=0.8106$ & 838.4162 & 1680.8 & 0.3831 \\
\hline
\end{tabular}




\begin{tabular}{|l|l|l|l|l|}
\hline Ge & $\hat{p}=0.8335$ & 841.1116 & 1684.2 & 0.8335 \\
\hline ZTP & $\hat{\lambda}=5.9914$ & 996.0346 & 1994.1 & 0.4720 \\
\hline DPL & $\hat{\theta}=0.2940$ & 867.1458 & 1736.3 & 0.2693 \\
\hline
\end{tabular}

As we see from the results, the $\operatorname{GZTP}(q, \theta)$ model has an AIC value less than other models even less than the EDW model with having the three parameters. Further, the K-S value of the new model is better than that of other models, except the K-S value of the EDW. In discrete distributions, the K-S statistic is usually calculated without its p-value (see, e.g., Nekoukhou and Bidram, [16]; Almalki and Nadarajah, [20]; Chakraborty and Chakraborty, [21]). Indeed, a less K-S value indicates a better fit among others. Empirical cdf plots for the fitted models are given in Figure 5.

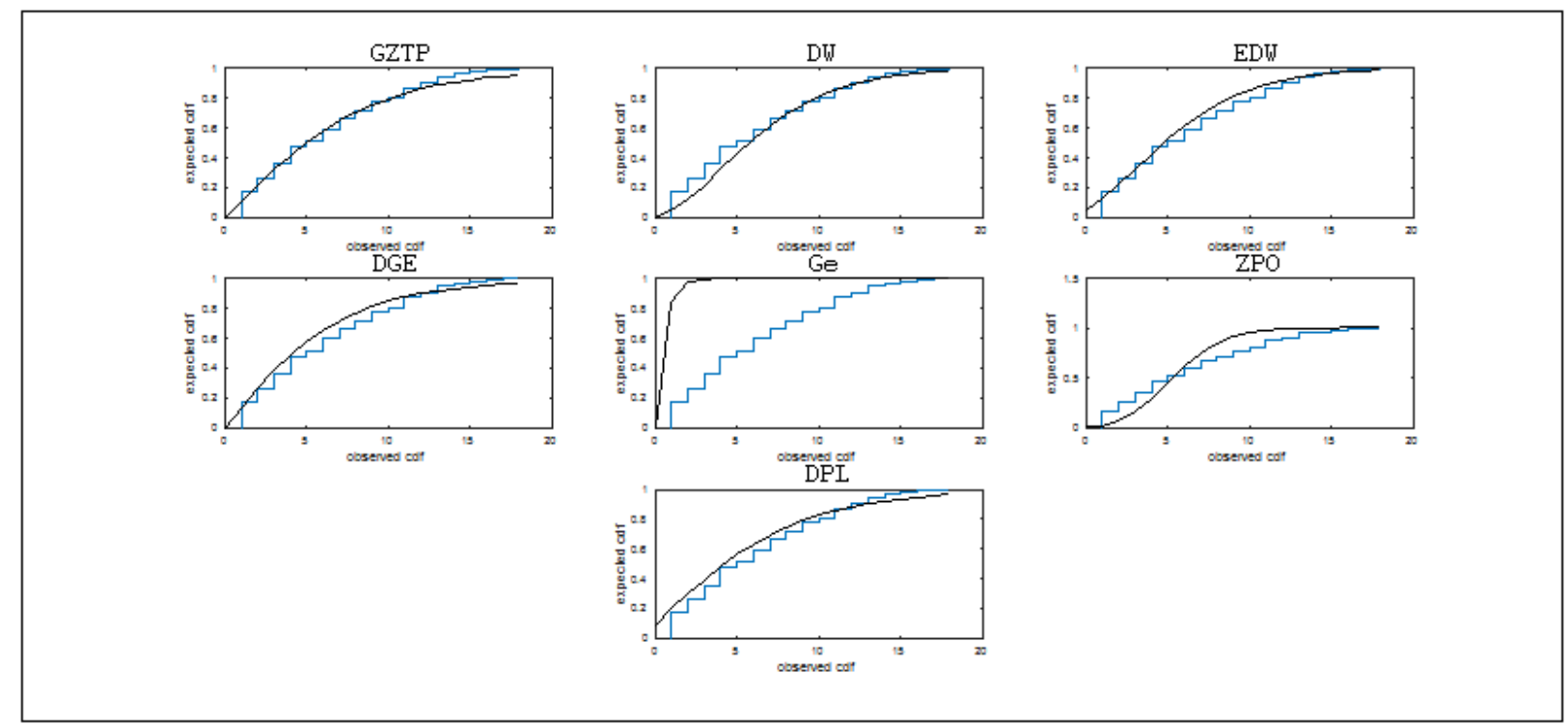

Figure 4. The first data: Empirical cdf plots for the fitted models

2. Second real data set: The data are rank frequencies of graphemes in Slovene language given in Table 3 of Makcutek [22] and have been also analyzed by Nekoukhou et al. [17]. The data are given in Table 9.

Table 9. Rank frequencies of graphemes in Slovene language

\begin{tabular}{|l|l|l|l|l|l|l|l|l|l|}
\hline$i$ & $f(i)$ & $i$ & $f(i)$ & $i$ & $f(i)$ & $i$ & $f(i)$ & $i$ & $f(i)$ \\
\hline 1 & 32036 & 6 & 16088 & 11 & 13034 & 16 & 7446 & 21 & 2606 \\
\hline 2 & 31891 & 7 & 16084 & 12 & 10517 & 17 & 6413 & 22 & 2554 \\
\hline 3 & 31122 & 8 & 15221 & 13 & 10514 & 18 & 5361 & 23 & 2463 \\
\hline 4 & 27150 & 9 & 14668 & 14 & 10216 & 19 & 5055 & 24 & 1675 \\
\hline 5 & 22905 & 10 & 14043 & 15 & 9568 & 20 & 4608 & 25 & 497 \\
\hline & & & & & & & & & $\mathrm{N}=313735$ \\
\hline
\end{tabular}

The results are given in Table 10. The AIC values and Figure 6 indicate that $\operatorname{GZTP}(q, \theta)$ model has a better fit than other models. Further, the K-S value of the new model is better than that of other models, except the K-S value of the EDW. Finally, using the first and the second data sets, we conclude that the proposed model works well in application, especially in modelling discrete data. 
Table 10. MLE, maximized $\ell, A I C$, and K-S values of the fitted models for the second data set

\begin{tabular}{|l|l|l|l|l|}
\hline Model & MLE & $-\ell(\hat{\boldsymbol{\theta}})$ & AIC & K-S \\
\hline GZTP & $\hat{q}=0.8378, \quad \hat{\theta}=0.3228$ & $9.3114 \times 105$ & $18623 \times 106$ & 0.0960 \\
\hline DW & $\hat{p}=0.9626, \quad \hat{\alpha}=1.4739$ & $9.4503 \times 105$ & $1.8901 \times 106$ & 0.1005 \\
\hline EDW & $\hat{p}=0.8517, \quad \hat{\alpha}=1.0407, \hat{\gamma}=1.9099$ & $9.4394 \times 105$ & $1.8879 \times 106$ & 0.0887 \\
\hline DGE & $\hat{\alpha}=1.3950, \quad \hat{p}=0.8808$ & $9.4396 \times 105$ & $1.8879 \times 106$ & 0.2858 \\
\hline Ge & $\hat{p}=0.8715$ & $9.3638 \times 105$ & $1.8728 \times 106$ & 0.8814 \\
\hline ZTP & $\hat{\lambda}=7.7787$ & $9.2318 \times 106$ & $2.4635 \times 106$ & 0.5062 \\
\hline DPL & $\hat{\theta}=0.2940$ & $9.5294 \times 106$ & $1.9059 \times 106$ & 0.3060 \\
\hline
\end{tabular}

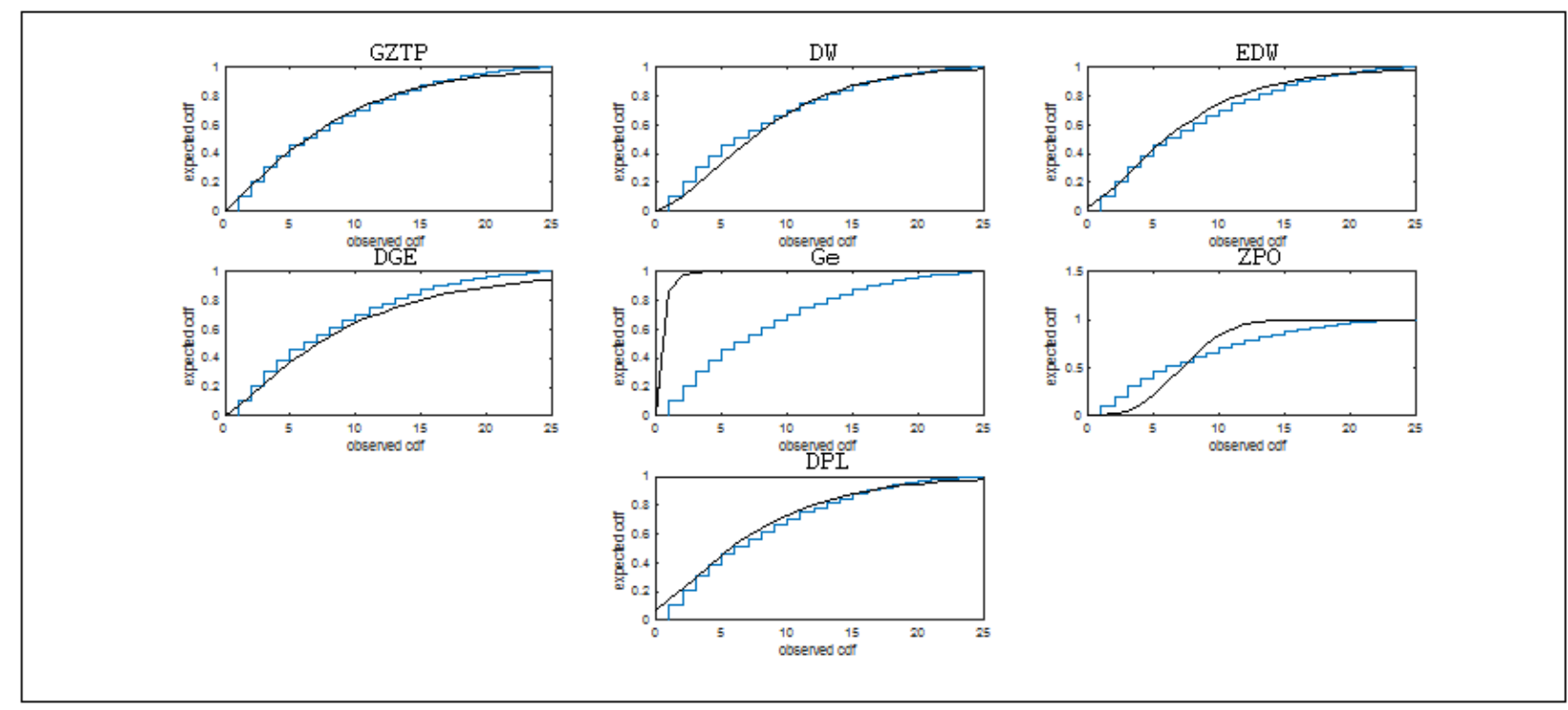

Figure 5. The second data: Empirical cdf plots for the fitted models

\section{CONCLUDING REMARKS}

In this paper, a new two-parameter discrete model with an increasing hazard rate function is introduced. The new model is obtained by compounding a geometric distribution with a zero-truncated Poisson distribution with a simple structure. In fact, the new model is obtained by considering maximum of $N$ iid geometric random variables, where $N$ has a zero-truncated Poisson distribution, with applications in parallel discrete systems. The basic statistical and mathematical properties are studied in this paper. Potentiality of the new model is indicated with the good results using the two real data sets. To complete this work, one can consider the minimum of the geometric random variables with applications in series discrete systems in reliability.

\section{CONFLICTS OF INTEREST}

No conflict of interest was declared by the authors.

\section{REFERENCES}

[1] Adamidis, K., Loukas, S., "A lifetime distribution with decreasing failure rate", Statistics and Probability Letters, 39: 35-42, (1998). 
[2] Barreto-Souza, W., Morais, A.L., Cordeiro, G.M., "The Weibull-geometric distribution." Journal of Statistical Computation and Simulation, 81(5): 645-657, (2011).

[3] Hemmati, F., Khorram, E., Rezakhah, S., "A new three-parameter ageing distribution", Journal of Statistical Planning and Inference, 141: 2266-2275, (2011).

[4] Lu, W., Shi, D., "A new compounding life distribution: The Weibull-Poisson distribution." Journal of Applied Statistics, 39: 21-38, (2012).

[5] Kuş, C., "A new lifetime distribution", Computational Statistics and Data Analysis, 51: 44974509, (2007).

[6] Tahmasbi, R., Rezaei, S., "A two-parameter lifetime distribution with decreasing failure rate." Computational Statistics and Data Analysis, 52: 3889-3901, (2008).

[7] Kemp, A.W., "Classes of discrete lifetime distributions", Communications in Statistics-Theory and Methods 33: 3069-3093, (2004).

[8] Shafaei N.M., Rezaei, R.A.H., Mohtashami, B.G.R., "Some discrete lifetime distributions with bathtub-shaped hazard rate functions", Quality Engineering, 25: 225-236, (2013).

[9] Akdoğan, Y., Kuş, C., Asgharzadeh, A., Kınacı, I., Sharafi, F., "Uniform-geometric distribution." Journal of Statistical Computation and Simulation, 86(9): 1754-1770, (2016).

[10] Gomez - Deniz, E., "A new discrete distribution: properties and applications in medical care", Journal of Applied Statistics, 40: 2760-2770, (2013).

[11] Nakagawa, T., Zhao, X., "Optimization Problems of a Parallel System with a Random Number of Units", IEEE Transactions on Reliability, 61: 543-548, (2012).

[12] Keilson, J., H. Gerber, H., "Some results for discrete unimodality." Journal of the American Statistical Association, 66: 386-389, (1971).

[13] Braden, B., "Calculating sums of infinite series", The American Mathematical Monthly, 99(7): 649655, (1992).

[14] Ferguson, T.S., A course in large sample theory, London: Chapman and Hall. (1996).

[15] Nakagawa, T., Osaki, S., 1975. "The discrete Weibull distribution", IEEE Transactions on Reliability, 24: 300-301, (1975).

[16] Nekoukhou, V., H. Bidram, H., "The exponentiated discrete Weibull distribution", SORT, 39: 127146, (2015).

[17] Nekoukhou, V., Alamatsaz, M.H., Bidram. H., "A discrete analogue of the generalized exponential distribution", Comm. Statist. Theory Methods, 41: 2000-2013, (2012).

[18] Sankaran, M., “The discrete Poisson-Lindley distribution”, Biometrics, 26, 145-149, (1970).

[19] Xie, M., Lai, C.D., "Reliability analysis using an additive Weibull model with bathtub-shaped failure rate function", Reliability Engineering and System Safety, 52: 87-93, (1995).

[20] Almalki, S.J., Nadarajah, S., "A new discrete modified Weibull distribution", IEEE 
Transactions on Reliability, 63, 68-80, (2014).

[21] Chakraborty, S., Chakraborty, D., "Discrete gamma distributions: Properties and parameter estimations", Communications in StatisticsTheory and Methods, 41, 3301-3324, (2012).

[22] Makcutek, J., "A generalization of the geometric distribution and its application in quantitative linguistics", Romanian Rep. Phys, 60: 501-509, (2008). 